Voix et Images

volxetimages

\title{
Les monologues de Sol : une initiation à la langue-Moi
}

\section{Laure Hesbois}

Volume 7, numéro 1, automne 1981

\section{Adrien Thério}

URI : https://id.erudit.org/iderudit/200307ar

DOI : https://doi.org/10.7202/200307ar

Aller au sommaire du numéro

\section{Éditeur(s)}

Les Presses de l'Université du Québec

\section{ISSN}

0318-9201 (imprimé)

1705-933X (numérique)

Découvrir la revue

\section{Citer cet article}

Hesbois, L. (1981). Les monologues de Sol : une initiation à la langue-Moi. Voix et Images, 7(1), 119-129. https://doi.org/10.7202/200307ar d'utilisation que vous pouvez consulter en ligne.

https://apropos.erudit.org/fr/usagers/politique-dutilisation/ 


\title{
Les monologues de Sol: une initiation à la langue-Moi
}

\author{
par Laure Hesbois
}

J'ai rencontré Sol en octobre 1979, à la sortie d'un spectacle triomphal au Théâtre de la Ville, à Paris. Il dédicaçait ses monologues (Rien détonnant avec $S o \eta^{1}$ et, en lui tendant mon exemplaire, j'ai été saisie d'un brusque accès de vanité et je n'ai pu résister au plaisir de lui annoncer que je venais d'entreprendre une étude sur les jeux de langage et que je comptais bien utiliser, pour ce travail, quelques-unes de ses merveilleuses trouvailles. Un instant $j$ 'ai même été tentée de dire vermouilleuse mais je me suis, Dieu merci, ressaisie à temps! Sol $\mathrm{m}$ 'a fort poliment écoutée puis, avec un sourire d'excuse parfaitement imité, il $\mathrm{m}$ 'a déclaré que les linguistes étaient en général des gens beaucoup trop savants pour lui et que c'était pour cela, sans doute, qu'ils avaient tant de peine à comprendre ses inventions.

En sortant de là, je brûlais d'envie de relever le défi et de tenter, une fois de plus ${ }^{2}$, de percer le secret de cet idiome inimitable, a la fois si limpide et si déconcertant. Cependant, rendue circonspecte (et modeste) par son commentaire, j'ai décidé de mettre de côté le pompeux appareil «scientifique * dont je m'étais servie jusqu'ici et dont j'avais d'ailleurs pu, en maintes occasions, constater les insuffisances pour retrouver, autant que possible, l'attitude du spectateur naîf qui, sans s'encombrer de théories, se laisse tout simplement prendre au charme d'une parole (d)étonnante.

J'ai donc choisi, comme point de départ le plus sûr, le plaisir que me procurent les performances linguistiques de Sol et je me propose d'en rendre compte le plus humblement possible, en m'appuyant d'abord et avant tout sur l'évidence sensible et en limitant mes interventions cartésiennes (on ne se défait pas d'un seul coup de ses mauvaises habitudes !) au minimum dont se satisfait généralement le sens commun.

La première constatation qui s'impose, c'est que la façon de parler de Sol fait partie d'un jeu d'illusion au second degré. Dès son entrée en scène il ne peut échapper à personne que le comédien Marc Favreau joue le rôle de Sol. L'artifice théâtral s'affiche ostensiblement à travers le maquillage outrancier, l'accoutrement burlesque, la mimique excessive qui accompagne 
chacun de ses propos. Mais il est tout aussi évident que le personnage ainsi composé ne représente rien de plus qu'une figure de cirque, prête à exécuter son propre numéro. Au terme de ce premier déguisement, Sol apparaît comme un concentré vivant de tous les amuseurs publics, clowns, saltimbanques, mimes, illusionnistes, etc., simultanément mobilisés, avec toutes les ressources de leur art respectif (culbutes, cabrioles, jongleries, escamotage, etc.). pour représenter un pauvre diable sans défense, nommé Sol, avec qui il faut bien se garder de le(s) confondre. Si Marc Favreau joue à être Sol (jusqu'à ne faire qu'un avec lui, nous assure-t-il dans la Préface, mais cette déMARCation n'est pas de notre compétence) Sol de son côté s'amuse à être "le pôvre Sol» sans jamais coïncider entièrement avec sa création. C'est ce clivage initial, ce décollage constant entre deux niveaux de représentation, qui confère au jeu de Sol sa véritable signification et qui explique le ton très particulier, tour à tour satironique et pitoyeux, du personnage protéiforme incarné par Marc Favreau.

A s'en tenir à la lettre du texte - et du spectacle - il semblerait que le pôvre sol s'identifie à l'un de ces malchanceux congénitaux, irrémédiablement voués à avoir le dessous, un de ces misérables besogneux qui arrivent tout juste à gagner leur petit pain. tantôt victimes des exployeurs et tantôt réduits au chômage, condamnés à attendre des semelles et des semelles devant le bourreau de placement; un de ces éternels inadaptés, pitoyables et grotesques, constamment étonnés de ce qui leur arrive, terrorisés par une civilisation écrasante (avec ses camionstres très énormes) persécutés par une administration délirante, abusés par un jeu politique insensé dont ils font immanquablement les frais. Plus précisément, une de ces victimes héréditaires façonnées par la grande noire sceur, métamorphosée depuis peu en belle trop mince, sans que le sort des mal lotis s'en trouve véritablement amélioré. Et la langue qu'il parle est à première vue un reflet de celle des déshérités de tout bord: langue hésitante dont on n'a pas eu le loisir d'étudier les règles et dont on se sert gauchement pour parer au plus urgent. Langue menacée que l'on craint sans cesse de voir disparaître, parce que le garnement aura brusquement décidé de la mettre dans sa poche ou parce qu'on aura été contraint de la donner au chat, ou éventuellement à la chatte, mais quelle différence cela peut-il bien faire?...

Toutefois, ce n'est là qu'une face du personnage et l'apitoiement du spectateur s'accompagne en général d'un sourire complice parce qu'il se rend parfaitement compte que la naïveté du pôvre Sol fait partie d'un rôle et qu'elle sert en réalité de prétexte à une satire aussi pénétrante que subtile. En effet ce prétendu maladroit a l'art d'éviter les chutes et d'opérer de spectaculaires rétablissements. Semblable au funambule détournant à son profit les lois de la pesanteur. Sol parvient à tirer parti des situations les plus contraignantes: réduit au chômage il découvre aussitôt une planque confortable devant le bourreau de placement. Dépossédé de sa langue, il trouve malgré tout le moyen de dire son fait au garnement responsable et profite même de l'occasion pour assurer celui-ci de ses ressentiments distancés. Trop peu 
instruit pour comprendre les grands principes de la politique nationale ou internationale, il n'en perçoit que mieux les basses manœuvres: expropriation des indigents de la Baie James, impérialisme économique des États-munis, dilapidation des ressources mondiales, prolifération des organismes inutiles, coups d'État militaires, etc. Et cette complicité tourne très vite à l'émerveillement. Car Sol, devenu magicien (le manteau rapiécé du clochard peut avoir des envolées de grand style), opère sous nos yeux des miracles. Humilié depuis sa naissance il adresse un fraternel hommage à la dignité du fier monde. Manceuvré de mille et une façons, il vole au secours d'une pauvre petite Marie honnête et lui indique le meilleur moyen de couper ses ficelles. Exposé sans défense à tous les dangers de la cruelle il n'hésite pas à recueillir la poubelle esseulée qu'il entend sauver du désespoir et de la rouille, sans compter les chatpardeurs et les polissons. Du fond de ses poches vides, le déshérité magnifique fait surgir des trésors d'humour et de tendresse. Avec ses deux mains gauches le bon à rien inspiré exécute en quatre phrases le plus extraordinaire des tableaux jamais peint, faisant jaillir sous nos yeux éblouis une explosion de couleurs folles, illuminant notre morne existence d'un instant de poésie pure.

Bref il est clair que le pôvre Sol dispose contre la malchance, la misère et I'humiliation, de ressources inépuisables, au premier rang desquelles il convient de placer ses prodigieuses performances verbales. Tout le jeu que j'ai tenté d'évoquer jusqu'ici se fonde sur une série de métamorphoses. Je pense que le même principe s'applique au langage et que la parole de Sol se caractérise d'abord et avant tout par l'équilibre instable de ses composantes, ce pourquoi sans doute elle est si difficilement accessible aux linguistes, qui s'acharnent à la fixer en une formule définitive.

Et, ici, une deuxième constatation s'impose: le langage de Sol est radicalement différent du nôtre et pourtant il nous est directement accessible, ce qui revient à dire que, s'il ne se conforme pas toujours aux normes en vigueur, il fonctionne cependant selon des règles précises, qui ne nous sont pas inconnues.

Rien qu'à entendre parler Sol, le moins instruit des spectateurs n'a aucune peine à se rendre compte que celui-ci éprouve des difficultés à coordonner l'articulation orale d'un mot et sa représentation graphique, en d'autres mots qu'il n'est pas très sûr de son orthographe ni de sa prononciation. Rien de surprenant d'ailleurs: Sol n'est pas très instructionné. II n'a pas appris grand chose à l'école de la grande noire sceur, excepté le cachottisme. Que l'orthographe française soit une des plus compliquées et des plus illogiques qui soient, chacun d'entre nous en a fait dans son enfance la douloureuse expérience et nul ne s'attriste particulièrement d'en voir transgresser les règles inflexibles. C'est donc avec une réelle sympathie (nuancée parfois d'un agréable sentiment de supériorité) que le spectateur assiste aux efforts infructueux du pauvre Sol, trébuchant parmi les doubles consonnes, les sons jumelés, les lettres superflues, etc. Et sa satisfaction ne fait que croittre lorsqu'il découvre 
que les erreurs en question sont en fait savamment calculées pour mettre en évidence les déficiences les plus flagrantes du système:

- deux lettres pour un seul son comme dans «pauvre» que Sol écrit plus ou moins phonétiquement, $p-0-v-r-e$ et qu'il prononce [p $v v r$;

- une seule lettre pour deux sons, soit $x=[k s]$ délibérément confondu par Sol avec [sk]. Ex. : «klinex»qu'il prononce [klinesk] et qu'il transcrit $k-I-i-n-e-s-q-u-e$ et, vice-versa, «gigantesque» transformé en [zigãteks] et graphiquement rendu par g-i-g-a-n-t-e-x-e;

- lettres superflues; ex. : «la pluspart» que Sol s'applique à prononcer [laplyspar] et qu'il écrit à cet effet l-a-p-I-u-s-s-p-a-r-t;

- différentes graphies d'un son identique [ã]=en, comme dans - «inventable $=$ qui reste à inventer et an comme dans «invantable» =qui ne peut être vanté, homonymes que Sol s'ingénie à faire coïncider;

- graphie identique de sons différents. Ex. : $x$ correspondant tantôt à [ks ] comme dans "excuse» et tantôt à [s] comme dans "dix». On ne s'étonnera pas de voir Sol confondre régulièrement les deux et de l'entendre présenter des esscuses à son institutrixte.

Toutefois cela ne suffit pas à expliquer la jubilation que nous procurent les hasardeuses transpositions auxquelles il se livre, même en tenant compte du fait que la maîtrise de l'orthographe est un privilège de classe, lié à tout un ordre de valeurs bourgeoises, auquel les miséreux de son espèce n'ont pas accès. En fait, ce que manifestent clairement les fautes de Sol, c'est l'écart entre les besoins réels de l'usager et les limitations d'un code, arbitrairement modelé sur l'usage d'un petit groupe. En jouant des divergences entre code oral et code graphique, Sol parvient à exprimer certaines nuances que ni l'un ni l'autre pris séparément ne permettrait d'enregistrer. Ainsi, en s'autorisant de la graphie aberrante pour justifier une prononciation fantaisiste, il produit un vocable inédit, travaller, qui par sa configuration sonore et sa ressemblance avec des mots comme avaler, cavaler, etc., ajoute à l'idée de atravail» une touche de précipitation et d'affolement particulièrement savoureuses. La transcription capricieuse du son [0] (o, au, eau, etc.) fournit à Sol le moyen d'enregistrer, si imparfaitement que ce soit, un fait d'intonation que l'orthographe usuelle néglige. En effet l'accent circonflexe de pôvre n'a pas pour fonction d'indiquer une prononciation fermée du o (Sol prononce [povr]) mais de souligner discrètement l'importance affective du vocable. II équivaut à un accent d'insistance. La «faute» ne doit donc pas être interprétée comme ignorance du code, mais bien plutôt comme un détournement de celui-ci à des fins expressives. Il en va de même de tous les émouvants ratages fondés sur la suppression, l'adjonction ou la permutation des syllabes, qui correspondent en général à un état de surexcitation et qui n'ont d'autre rôle que de traduire le trouble du personnage. C'est l'intensité de sa surprise qui lui fait escamoter une syllabe dans extraordinaire (esstradinaire). C'est un 
éblouissement temporaire, résultant du désir comblé, qui l'amène à confondre «merveille» et «vermeil» en vermeilleux qui, combiné aux manifestations physiologiques du plaisir (à savoir une augmentation sensible des sécrétions) produit finalement l'un des vocables les plus typiques de son idiolecte, vermouilleux.

Enfin, certaines erreurs ont pour but de révéler les associations secrètes que nous établissons spontanément entre divers termes en fonction de leur analogie phonétique. En gratifiant essentiel d'un c à la place du t (essencien) Sol avoue le lien qui, pour les esprits simples, rattache au ciel toutes les questions importantes. La simple adjonction d'un t à chapardeurs (chatpardeurs) lui permet d'établir une incontestable parenté entre les maraudeurs et les chats errants, entre la ruse féline des uns et la souplesse furtive des autres.

Bref, tout en confirmant l'impression d'infériorité (sociale et mentale) que le personnage s'applique à créer, les fautes permettent à Sol de dénoncer les défaillances de la langue dite correcte et de faire passer, en plus du sens avoué, un message secret que nous (je veux dire les honnêtes gens, pas les linguistes) n'avons aucune peine à déchiffrer, bien qu'il ne soit pas officiellement codé.

Mais il n'y a pas que l'orthographe qui puisse empoisonner l'existence des gens peu instruits. II y a aussi le dictionnaire et ses redoutables arrêts. C'est lui en effet qui, en fonction de critères impénétrables au commun des mortels, accorde ou refuse le droit de cité aux mots, lui qui tranche si telle ou telle forme est recevable ou non, sans se préoccuper ni des besoins de l'usager, ni des solutions qu'il est capable d'inventer. Sol ignore naturellement les décisions de l'Académie et il se contente de fabriquer au fur et à mesure les mots dont il a besoin, en recourant au procédé le plus simple, c'est-à-dire la dérivation. Et comme il n'a pas le loisir d'en étudier tous les raffinements, il se contente d'exploiter deux ou trois formules particulièrement rentables à savoir:

1. Une formule élémentaire qui permet de fabriquer des verbes faciles à conjuguer - à partir de noms très courants, par simple adjonction du siffixe -er et redoublement accessoire de la consonne; ex.: d'après friction, frictionner on aura émotion - émotionner, palpitation - palpitationner, admiration - admirationner, «occupassion» - occupassionner 3 , etc. Formule parfaitement orthodoxe, comme on voit, et qui passerait facilement inaperçue, n'était-ce l'usage excessif qu'en fait Sol, témoignant par là de sa prétendue simplicité.

2. Une formule tout aussi pratique, permettant d'obtenir des noms à partir de verbes, mais faisant intervenir un suffixe étranger -ing, ce qui est une façon discrète de dénoncer la constante agression dont est victime la langue française au Canada. Comme tous ses 
concitoyens Sol est condamné à faire du vagabonding, du promening, de l'échanging, etc.

3. Une formule personnelle qui consiste à refaire la plupart des verbes grâce à un suffixe absolument inédit, -ouiller, résultat probable d'une confusion entre des formes globales comme mouiller, brouiller, souiller, rouiller... et des formes composées comme ennu(i) -yer, balai -yer, etc.; quoi qu'il en soit, l'intérêt de ce pseudo-suffixe réside dans sa texture sonore, qui fait irrésistiblement penser au cri de douleur du maladroit qui vient de recevoir un coup sur les doigts. C'est une des particularités les plus frappantes du langage de Sol qui regretouille, s'énervouille, fracassouille, etc. mais qui ne veut pas que la poubelle désespérouille ${ }^{4}$.

Cependant, pour un esprit peu évolué, même le procédé le plus simple peut présenter quelques difficultés. Et le pôvre Sol ne parvient pas toujours à distinguer entre les formations logiques et les formations déviantes, ce qui l'entraîne notamment à fabriquer de réjouissants adverbes: montagneusement, tourbillonnement, essplosionnement, calumètement, scrutineusement, arc-en-cielement, etc. et vice versa, il lui arrive parfois d'interpréter les mots simples comme des composés et de traduire, par exemple, intellectuelle par non télectuelle, diplôme par double plome, cybernétique par superlativement bernétique ou encore si tant bernétique, pour la plus grande joie du spectateur qui profite de l'insolence candide du personnage à l'égard des experts, tout en savourant sa supériorité sur lui.

Ajoutons que le rôle du dictionnaire ne se borne pas à juger la forme mais qu'il décide également du sens des mots. Et que les risques d'erreur ne sont pas moins grands dans ce domaine. Tandis que les spécialistes se livrent à de savantes distinctions entre sens propre et sens figuré, sens large et sens restreint, etc., l'homme de la rue se contente en général d'un seul sens, le plus évident, le plus concret, ce qui l'amène souvent à interpréter "au pied de la lettre» des expressions dites figurées. Pour Sol, un «agent double» ne peut être qu'un agent deux fois plus gros que les autres et il trouve tout naturel d'appeler «porte-plumes» en parlant d'un indien, ce que d'autres appelleraient sa tête ou son chef. II se montre particulièrement sensible à l'analogie formelle et cherche à établir une corrélation sémantique envers les mots qui se ressemblent; c'est ainsi que l'inventable et l'invantable doivent nécessairement finir par se confondre et qu'un rejeton doit s'attendre à être rejeté...

Toutes ces violations mineures à l'encontre du dictionnaire procurent au spectateur le même type de satisfaction revancharde que les entorses à l'orthographe. Mais Sol ne s'arrête pas là. Dans sa confusion mentale il lui arrive fréquemment de ne pas savoir exactement comment on doit dire et de prendre ainsi un mot pour un autre ${ }^{5}$. Certes, dans ce cas, pour que la communication reste possible, il faut que l'auditeur soit en mesure d'opérer la rectification et donc que le contexte soit suffisamment explicite par ailleurs. 
Lorsque tel est le cas, il devient en quelque sorte complice d'une opération de contrebande visant à faire passer deux messages en un seul: ce que Sol prétend dire, ce qu'il dit effectivement. C'est un instrument de satire merveilleusement efficace. Même si l'on corrige dépités en députés, on n'aura jamais affaire qu'à des représentants du peuple un peu aigris. Le garnement restera toujours un gouvernement qui a mal tourné et le cachottisme une instruction religieuse entachée d'hypocrisie. Le vote à main armée ne peut être qu'une parodie de la démocratie, très proche du coup de force, et les médiocres d'information une entreprise d'abêtissement collectif. Aux yeux de Sol les ébats de la chambre manquent évidemment de sérieux et les bourreaux de placement lui inspirent une insurmontable frayeur. En revanche le fier monde suscite son admiration et la pauvre petite Marie-honnête redevient, grâce à lui, une personne respectable. Le lapsus produit au grand jour les sentiments que nous nous efforçons de cacher, les vérités que nous n'oserions formuler: Sol s'en sert pour signaler la voracité des classes digérantes, la morosité des sinistres, la vertu glacée des dames de la congélation, la lente usure des usagés du métro, la platitude des sornettes à la lune, etc.

On obtient des résultats à peu près identiques avec le calembour et Sol ne se prive pas non plus des avantages offerts par ce procédé populaire. C'est ainsi qu'il démontre que le cléricalisme est responsable de l'ignorance où a croupi la population québécoise sous Duplessis, en rebaptisant cette époque l'école de la grande noir sceur. C'est ainsi qu'il dénonce le génocide auquel la peur conduit les blancs en Afrique du Sud: cette clique broie du noir. Enfin c'est ainsi qu'il démarque les intérêts économiques qui se dissimulent sous les promesses humanitaires faites aux indigènes - indigents: "on est là parce qu'on veut votre bien (et on l'aura)".

Dans tous les exemples cités il est clair que le contenu (satirique) du message passé en contrebande joue un rôle important dans la satisfaction éprouvée par le spectateur. Mais il y a plus. L'équivoque possède en elle-même des vertus libératrices. Elle affranchit l'individu d'une des lois les plus tyranniques du langage en faisant la preuve qu'il est, en réalité, tout à fait possible de dire au moins deux choses à la fois. La preuve est aussi séduisante que celle fournie par l'acrobate pour démontrer qu'il est possible, et même facile, de marcher sur les mains. L'un et l'autre oublient seulement de préciser qu'il y faut beaucoup de talent. Ou beaucoup d'innocence. Avec Sol il est inutile d'essayer de départager l'une de l'autre.

Contrairement aux altérations morphologiques et aux glissements sémantiques signalés plus haut, qui laissaient intact le principe d'un code, c'est-à-dire d'une convention qui assigne univoquement tel contenu à telle expression, le message truqué et le calembour ouvrent une brèche dans le système. Ils laissent entrevoir la possibilité pour chaque individu de décider lui-même du rapport et de conférer à ce qu'il dit ou à ce qu'il entend, en plus du sens officiel (ou à la place de) un sens individuel, satisfaisant ainsi un vieux rêve enfoui en chacun d'entre nous, celui d'une langue sur mesure, calquée 
sur nos exigences individuelles, qu'un autre rêveur appelle justement la langue-Moi ${ }^{6}$. Ce désir reçoit sa satisfaction presque complète grâce à un autre type de formation extrêmement fréquent chez Sol et que les spectateurs instruits identifieront immédiatement avec les mots-valises de Lewis Caroll et consorts. Mais les plus ignorants n'auront aucune peine à en saisir le principe et n'en apprécieront pas moins l'efficacité. Ayant remarqué que le pôvre Sol est un être extrêmement craintif, ils comprendront fort bien qu'aucun des termes courants (épouvantable, effroyable) ne suffit à tradụire exactement l'intensité de sa frayeur et que ce n'est qu'en combinant ceux-ci qu'il peut nous en donner une juste idée. Et ils admettront de bonne grâce que, si le terme épouffroyable n'existe pas, il convenait effectivement de l'inventer! Ils reconnaîtront la souplesse du procédé qui permet de nuancer l'expression de nos sentiments et de présenter les êtres et les choses sous le jour exact qui nous convient. Comment désigner plus justement que par camionstres ces énormes choses grondantes, surgissant à l'improviste pour vous happer sous leurs roues? Comment rendre autrement que par terriblifique ce subtil mélange d'admiration et de crainte que nous inspirent certains spectacles? Et comment présenter de façon plus économique et plus éloquente un écrivain vaniteux, un critique hypocrite, un employeur qui vous exploite, qu'avec les termes écrivaniteux, hypocritique, exployeur. Sol lui-même se qualifie de satironique, et cet adjectif lui va à merveille. Les associations les plus saugrenues trouvent leur justification dans l'incontestable congruence sonore des vocables mis en jeu. Et Sol a l'art des rapprochements qui surprennent et séduisent à la fois, autrement dit (d)étonnent, par exemple entre les miracles de la science et les mystères divins (l'électrinité), entre la civilisation et l'asservissement (la servilisation) entre la célébrité et les dons de l'alcool (la célébriété), entre les négociations politiques et les mégots de cigarettes /la mégociation), entre le triste sort d'une poubelle en tôle, exposée aux intempéries, et celui d'une pauvre gosse abandonnée au coin de la rue et qui désespérouille. Grâce à ses propres performances, Sol livre au spectateur un procédé fort simple, permettant à chacun de se créer, à peu de frais, un vocabulaire d'une originalité garantie et néanmoins accessible aux autres : il suffit de tailler, dans le tissu du langage commun, des morceaux à peu près satisfaisants, et de les assembler ensuite à sa manière (bord à bord, l'un sur l'autre, l'un dans l'autre, peu importe) ${ }^{7}$ pour composer un mot inédit, parfaitement approprié à la situation ${ }^{8}$. C'est entre autres un excellent moyen de résoudre les contradictions qui embarrassent tant les usagers respectueux du code. Avec Sol, un imperméable qui laisse passer l'eau (et l'expérience suffit à prouver que c'est là une mésaventure assez fréquente) est tout simplement rébaptisé impermouillable. Et quand la publicité se trouve dans l'impossibilité absolue de vanter un produit (invantable, puisque inventable) il lui cnseille de se publicitaire, une fois pour toutes. Enfin cette sorte de patchwork lexical présente cet avantage supplémentaire de pouvoir indifféremment passer pour un rapiéçage de fortune, imposé par la disette, ou pour une manifestation de génie créateur. Sol le clochard et Sol l'artiste y trouvent également leur compte et le spectateur ne peut que se réjouir d'assister une fois de plus à une métamorphose. 
Le procédé s'applique avec un égal succès aux locutions idiomatiques et aux clichés de toutes espèces qui constituent la trame de nos conversations quotidiennes. Quand il ne les prend pas au pied de la lettre ${ }^{9}$, Sol les corrige ou les combine pour les accommoder à ses besoins. Tout Canadien français a appris dès sa plus tendre enfance que la révolte contre l'ordre social est un péché et que «quiconque est né pour un petit pain doit s'en accommoder". D'autre part, tout prolétaire sait ce qu'il en coûte de gagner son pain. Sol appartient à l'une et l'autre de ces catégories et c'est pourquoi il s'inquiète. à juste titre, de la façon dont il doit s'y prendre pour arriver à gagner son petit pain. II sait qu'il est impoli de couper la parole à quelqu'un, mais il est naturellement encore plus choqué lorsqu'il s'agit d'un missionnaire à qui l'on ose couper la bonne parole.

C'est avec ces modestes créations artisanales que Sol démontre le plus magistralement les extraordinaires pouvoirs de son Verbe, capable de reproduire à son échelle le mystère de la Sainte Trinité, c'est-à-dire de surmonter l'opposition du Même et de l'Autre. de réaliser l'unité consubstantielle de deux ou plusieurs vocables distincts. Ce sont elles également qui marquent la limite des distorsions permises si l'on tient à éviter le charabia ${ }^{10}$. Chacun sait qu'au cirque les risques sont calculés au millimètre et à la seconde près. Et Sol ne fait pas exception à cette règle. À cet égard il est intéressant de remarquer que, mises à part quelques fautes de conjugaison (en particulier en ce qui concerne l'emploi des auxiliaires être et avoir dans la formation du passé composé) la construction des phrases peut passer pour à peu près normale. La seule exception notable consiste dans l'accumulation des intensifs (si, tout, tellement, etc.) qui convient tout à fait à l'étonnement naïf du spectateur. En somme, si déconcertant qu'il puisse paraître, le langage de Sol reste parfaitement adapté à la communication. II s'agit toujours d'un écart soigneusement contrôlé. Et c'est là encore une source de plaisir appréciable pour le spectateur: frôler à chaque instant le plongeon mortel, avec la garantie qu'il ne se produira pas. Avec en prime la satisfaction de se sentir supérieur à celui qui exécute l'exercice périlleux.

Car enfin, tous les exploits de Sol ont pour point de départ simulé l'ignorance. Sol ignore que langue parlée et langue écrite sont deux manifestations différentes du langage qui ne coïncident que très grossièrement. II ignore que certaines formes, au demeurant tout à fait logiques, sont arbitrairement déclarées irrecevables et que le sens d'un mot peut varier considérablement d'un contexte à l'autre. II ignore jusqu'à l'existence d'un code destiné à fixer de façon immuable le rapport entre telle expression et tel contenu. Résultat; il en est réduit à improviser, avec les ressources limitées dont il dispose, en fonction des exigences de la situation. Ce faisant, il invente une langue absolument inédite, plus concrète, plus souple, beaucoup mieux adaptée aux circonstances et à la personnalité du sujet. Du même coup il fait éclater les catégories mentales liées à une utilisation conventionnelles du langage et propose, à la place, un réseau de relations mouvantes, mettant en jeu les réactions individuelles du locuteur d'une part et la réalité matérielle du langage 
d'autre part. Tel me semble en fin de compte le secret du plaisir intense que nous procurent ce langage. Il s'agit, à la lettre, d'incantation, c'est-à-dire d'une formule spécialement conçue pour forcer la réalité à coïncider avec nos désirs mais, contrairement aux sorciers qui s'en réservent le privilège, Sol nous en livre généreusement la recette et pousse même l'élégance jusqu'à déguiser son don en une infirmité.

1. Rien d'étonnant avec Sol, Montréal, Stanké, 1978. Tous les exemples utilisés pour cette étude ont été tirés de ce recueil.

2. Ceci est en effet une récidive. Ma première tentative s'était soldée par un article intitulé Sol: une mise à l'épreuve du signe, publié par le Journal canadien de recherche sémiotique, vol. IV, $\mathrm{n}^{\circ} 2, \mathrm{pp}$. 41-53.

3. A noter la graphie fautive de ce terme qui suffit à établir une intéressante corrélation entre les activités d'une personne et ses passions.

4. On peut interpréter ce terme comme une variante originale de désespérer (désesperouiller) ou comme un composé obtenu à partir de désespérer + rouiller. Étant donné le contexte, c'est cette dernière interprétation qui me paraît la plus satisfaisante.

5. Titre d'une pièce bien connue de Jean Tardieu, dans laquelle l'auteur utilise systématiquement ce procédé. Ciel, mon zébu/ s'écrie la marquise adultère. Et tout le monde comprend qu'il ne peut évidemment s'agir que du mari jaloux.

6. Jean Tardieu, Préface de Le Professeur Froeppel, Paris, Gallimard, 1976.

... la Langue-Moi (la seule qui soit intégrale et irréductible) ou encore la Signification Universelle (chaque être, chaque objet de ce monde étant à fois son Existence et son Signe).

7. Techniquement les spécialistes distinguent volontiers deux types de formation:

- la formation "gigogne" qui consiste à articuler à la suite l'une de l'autre les diverses composantes, en profitant généralement d'une analogie phonétique. Ex.:
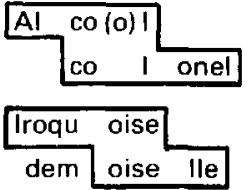

(Prévert)

(Queneau)

(Heine - cité par Freud.

Exemple canonique)

(Queneau)

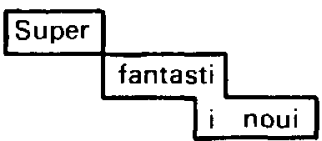


- la formation "sandwich» qui suppose l'insertion partielle ou totale d'une unité a l'intérieur d'une autre. Ex.:

ridi cu liser
co cu

(Rostand)

Les créations de Sol utilisent indifféremment l'un ou l'autre procédé mais ses découpages sont toujours d'une remarquable économie:

musul man ex ploi teur
men teur em plo(i) yeur

fran cophonie

am b assadeur

ca cophonie em b $r$ assade

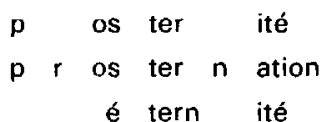

8. II suffit parfois d'une toute petite retouche: un simple préfixe permet de rectifier le nom d'un vieux meuble dont les tiroirs sont devenus difficiles à ouvrir et qui en bonne logique, devrait s'appeler une malcommode plutôt qu'une commode. La même recette vaut pour adapter une formule de politesse au destinataire: je sais que votre temps est déprécieux... déclare la belle trop mincé aux membres et membranes de l'assemblée paraplégilative.

9. Prendre une formule au pied de la lettre c'est privilégier le sens concret, c'est interpréter le mot comme équivalent symbolique de l'expérience et non comme valeur abstraite à l'intérieur d'un système. C'est donner le pas à la représentation sur la signification ou, en d'autres termes, faire passer l'adéquation du langage au réel avant le respect du code. C'est incontestablement répondre aux exigences de la langue-moi.

10. A partir du moment où les formes produites ne sont plus assimilables aux unités d'un code commun, il ne peut plus y avoir à proprement parler de communication. Tel ou tel assemblage sonore peut naturellement présenter certaines propriétés expressives, mais la perception et l'interprétation de celles-ci sont toujours aléaatoires. Dans ce cas il ne s'agit plus de communication mais de communion esthétique. 